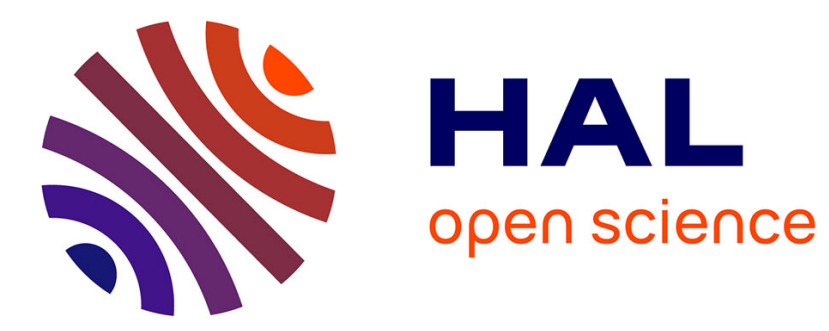

\title{
Examples of Fast and Slow Convergence of 2D Asynchronous Cellular Systems
}

\author{
Nazim A. Fatès, Lucas Gerin
}

\section{To cite this version:}

Nazim A. Fatès, Lucas Gerin. Examples of Fast and Slow Convergence of 2D Asynchronous Cellular Systems. Journal of Cellular Automata, 2009, 4 (4), pp.323-337. hal-00260184v4

\section{HAL Id: hal-00260184 \\ https://hal.science/hal-00260184v4}

Submitted on 31 Oct 2008

HAL is a multi-disciplinary open access archive for the deposit and dissemination of scientific research documents, whether they are published or not. The documents may come from teaching and research institutions in France or abroad, or from public or private research centers.
L'archive ouverte pluridisciplinaire HAL, est destinée au dépôt et à la diffusion de documents scientifiques de niveau recherche, publiés ou non, émanant des établissements d'enseignement et de recherche français ou étrangers, des laboratoires publics ou privés. 


\title{
Examples of Fast and Slow Convergence of 2D Asynchronous Cellular Systems
}

\author{
Nazim Fatès and Lucas Gerin
}

October 31, 2008

\begin{abstract}
This article studies the convergence properties of some 2D cellular automata, when a single cell is updated at random at each time step. We tackle this question for a particular set of rules, namely, the totalistic rules with nearest neighbours. We focus on a few examples that represent, in our view, the diversity of behaviours found in dimension two. These behaviours are analysed quantitatively with an estimation of the time needed to converge to a fixed point.
\end{abstract}

\section{Introduction}

The present paper is intended as a first step to understand the convergence properties of some 2D cellular automata subjected to a random perturbation. We focus on the fully asynchronous dynamics, where a single cell is uniformly sampled and updated at each time step. This scheme contrasts with the classical synchronous dynamics, where each cell is updated at each time step, and with the $\alpha$-asynchronous dynamics, where each cell has a probability $\alpha$ to be fired at each time unit.

So far, asynchronous cellular automata have been mainly studied experimentally. Among the first analytical results on asynchronous cellular automata, a new classification of rules was proposed in dimension 1 [3]. This approach uses the mean time needed to reach a fixed configuration as a discriminating factor. To our knowledge, the first analytical work related to two-dimensional asynchronous CA was carried out by Regnault et al. on the Minority rule [7]. Our purpose is to determine whether the classes found in $1 \mathrm{D}$ are also present in 2D, and to see if new classes appear. We select some totalistic rules that are, in our view, representative of the different behaviours found in two dimensions. 
One of our motivations for this work is to examine CA under the light of their robustness to asynchronous updating. To what extent does a random updating scheme change qualitative properties of a given system? For the sake of conciseness, we refer to $[3,6]$ for a review of works related to asynchronism and for a discussion on updating schemes. The different types of convergence we exhibit, from very fast to very slow, seem to confirm that the updating scheme is an important factor to understand the evolution of a cellular automaton.

\section{Definitions and Notations}

\subsection{Topology of the environment}

Let $\Lambda$ be the two-dimensional square grid of size $L$, with torical boundary conditions (i.e., we identify $\Lambda$ with $\mathbb{Z} / L . \mathbb{Z} \times \mathbb{Z} / L . \mathbb{Z}$ ). We denote by $n=L^{2}$ the total number of cells $; n$ is the central parameter that will be used to quantify the scaling properties of the systems.

For a cell $c$ and an integer $k$, we define the sphere $\partial B(c, k)$ as:

$$
\partial B(c, k)=\left\{c^{\prime} \in \Lambda \mid d\left(c, c^{\prime}\right)=k\right\},
$$

where $d$ is the usual graph distance on the torus. Below is a representation of the sphere $\partial B(c, 3)$, with $c$ in black and $L=10$ :

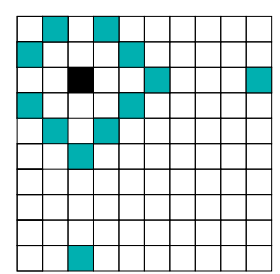

A configuration $\sigma=\left\{\sigma_{c}\right\}_{c \in \Lambda}$ is an association of each cell $c$ to a state $\sigma_{c}$ in $\{0,1\}$. We denote by $|\sigma|_{q}$ the number of occurrences of state $q$ in configuration $\sigma$. Configurations are modified at discrete times, according to a local transition function. The locality of the interactions between cells comes from the association to each cell $c \in \Lambda$ with its von Neumann neighbourhood: $\mathcal{N}(c)=\{c, c+\vec{n}, c-\vec{n}, c+\vec{e}, c-\vec{e}\}$, where $\vec{n}=(0,1)$ and $\vec{e}=(1,0)$.

\subsection{Asynchronous 2D Totalistic Rules}

We now define our cellular automaton as a discrete dynamical system. Each system is determined by a function $\phi:\{0,1\}^{5} \rightarrow\{0,1\}$ which governs the interaction of the cells of the grid. Such a function $\phi$ is called a local transition 
rule. In this paper, we consider only the totalistic rules, i.e., rules $\phi$ which can be written

$$
\phi\left(q_{1}, \ldots, q_{5}\right)=f\left(q_{1}+\cdots+q_{5}\right),
$$

where $f:\{0, \ldots, 5\} \rightarrow\{0,1\}$. There are 64 totalistic rules. We associate to each function $f$ the code $\mathbb{T} i$ where $i=f(0) \cdot 2^{0}+f(1) \cdot 2^{1}+\cdots+f(5) \cdot 2^{5}$. We also represent rules with a table, below is the table of $\mathbb{T} 32$ :

\begin{tabular}{|c||l|l|l|l|l|l|}
\hline$s$ & 0 & 1 & 2 & 3 & 4 & 5 \\
\hline$f(s)$ & 0 & 0 & 0 & 0 & 0 & 1 \\
\hline
\end{tabular}

Rule $\mathbb{T} 32$ stands for the rule which "leaves" a cell into the state 1 if and only if it is surrounded by all $1 \mathrm{~s}$.

We restrict our study to the fully asynchronous dynamics in which one single cell is updated at each time step. To define this type of asynchronism, we associate to each local rule $\phi$ its global updating rule $\Phi:\{0,1\}^{\Lambda} \times \mathcal{P}(\Lambda) \rightarrow$ $\{0,1\}^{\Lambda}$ where $\Phi(\sigma, \mathcal{U})$ is the configuration obtained by updating the cells in $\mathcal{U}$ in the configuration $\sigma$ according to the local rule $\phi$. More formally, if we write $\sigma^{\prime}=\Phi(\sigma, \mathcal{U})$ :

$$
\sigma_{c}^{\prime}= \begin{cases}f\left(\sigma_{c}+\sigma_{c+\vec{n}}+\sigma_{c-\vec{n}}+\sigma_{c+\vec{e}}+\sigma_{c-\vec{e}}\right) & \text { for } c \in \mathcal{U} \\ \sigma_{c} & \text { otherwise }\end{cases}
$$

A local rule $\phi$ and a sequence $\left(\mathcal{U}_{t}\right)_{t \in \mathbb{N}}$ of updates defines the sequence of configurations $\left(\sigma^{t}\right)_{t \in \mathbb{N}}$ :

$$
\begin{aligned}
& \sigma^{0} \in\{0,1\}^{\Lambda}, \\
& \sigma^{t+1}=\Phi\left(\sigma^{t}, \mathcal{U}_{t}\right), \text { for } t \in \mathbb{N} .
\end{aligned}
$$

The classical synchronous updating is defined with $\forall t, \mathcal{U}_{t}=\Lambda$. The fully asynchronous dynamics is obtained by taking $\left(\mathcal{U}_{t}\right)_{t \in \mathbb{N}}$ as a sequence of independent random variables $\left(U_{t}\right)_{t \in \mathbb{N}}$ that select one cell uniformly from $\Lambda$ :

$$
\left(\mathcal{U}_{1}, \mathcal{U}_{2}, \ldots\right)=\left(\left\{U_{1}\right\},\left\{U_{2}\right\}, \ldots\right) .
$$

The sequence $\left(\sigma^{t}\right)$ is then a discrete Markov chain with values in $\{0,1\}^{\Lambda}$.

\subsection{Convergence Time}

For a local rule $\phi$, we denote by $\mathfrak{F} \phi$ the set of the fixed points of the global rule $\Phi$ associated to $\phi$. It consists of the configurations that remain unchanged, whatever the cell selected for updating:

$$
\mathfrak{F} \phi=\left\{\sigma \in\{0,1\}^{\Lambda} \mid \text { for any cell } c \in \Lambda, \Phi(\sigma,\{c\})=\sigma\right\} .
$$


Note that for a given $\phi$, the sets of fixed points under synchronous and asynchronous updating are identical.

Definition 1. For a given function $\phi$ and an initial configuration $\sigma^{0}$, let $T_{\phi}\left(\sigma^{0}\right)$ be the time of convergence of the sequence $\left(\sigma^{t}\right)_{t \in \mathbb{N}}$, that is, the random variable:

$$
T_{\phi}\left(\sigma^{0}\right)=\min \left\{t \in \mathbb{N} \mid \sigma^{t} \in \mathfrak{F} \phi\right\},
$$

with $\min \emptyset=+\infty$. The Worst Expected Convergence Time (WECT) of rule $\phi$ is given by:

$$
\mathrm{WECT}_{\phi}(n)=\max _{\sigma^{0}} \mathbb{E}\left[T_{\phi}\left(\sigma^{0}\right)\right]
$$

We list three reasons for which we are interested in this quantification:

1. In dimension one, Fatès et al. [3] have studied the WECT of the Elementary Cellular Automata with two quiescent states. This work revealed that the asymptotic behaviour of $\mathrm{WECT}_{f}(n)$ provides a novel classification of the 1D cellular automata. Precisely, they have shown that these rules may be classified into 5 families, according to whether $\mathrm{WECT}_{f}(n)$ is $\Theta(n \log n), \Theta\left(n^{2}\right), \Theta\left(n^{3}\right), \Theta\left(n 2^{n}\right)$ or infinite ${ }^{1}$.

2. Another motivation comes from algorithmic complexity theory, since cellular automata are often thought as model in computability theory. With this point of view, it is natural to ask what happens when the system starts from the "worse" configuration.

3. Alternatively, if we think of cellular automata as models of physical or biological systems, studying the WECT provides us with an estimation of the maximum time needed to go back to equilibrium when a perturbation is applied.

\subsection{The results}

We recover in dimension two some of the classes met in dimension one, as well as a new one: $\approx n^{3 / 2}$. We have given to each of these rules a name to facilitate their identification; this name is chosen after their global behaviour. The five different behaviours we study are summarised in table below:

\footnotetext{
${ }^{1}$ We write $f_{n}=\Theta\left(g_{n}\right)$ when there exist two positive numbers $C^{-}, C^{+}$such that, for $n$ large enough, $C^{-} g_{n} \leq f_{n} \leq C^{+} g_{n}$.
} 


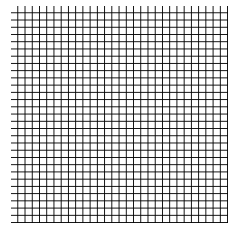

$t=0$

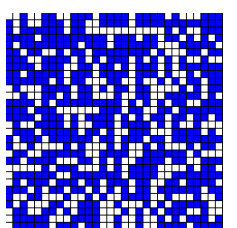

$t=n$

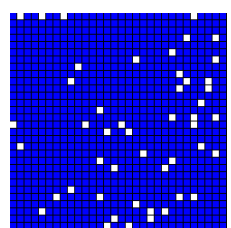

$t=3 n$

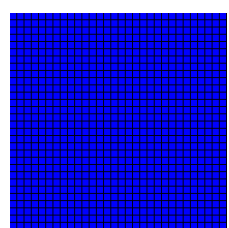

$t=7 n$

Figure 1: Example of a simulation for the coupon collector rule $\mathbb{T} 63$. Cells in state 1 (resp. 0) are coloured in black (resp. white), a convention which is kept throughout the paper. Simulations were obtained with the FiatLux CA simulator [2].

\begin{tabular}{|l|c|l|l|}
\hline Name & $\phi$ & $\mathrm{WECT}_{\phi}(n)$ & Observed behaviour \\
\hline Coupon collector & $\mathbb{T} 63$ & $\Theta(n \log n)$ & Very fast convergence to $1^{\Lambda}$ \\
Epidemic & $\mathbb{T} 62$ & $\approx n^{3 / 2}$ & Fast convergence to $1^{\Lambda}$ \\
Majority & $\mathbb{T} 56$ & $\Theta\left(n^{2}\right)$ & Fast convergence \\
Erratic & $\mathbb{T} 10$ & $>\lambda^{n}$ (conj.) & Slow convergence (metastability) \\
Parity Counter & $\mathbb{T} 21$ & infinite & Noise-like evolution \\
\hline
\end{tabular}

\section{Fast convergence: polynomial times}

In this section, we study three rules which converge in polynomial time, i.e., the scaling of their worst expected convergence time is less than a polynomial in $n$. These three rules are presented from the quickest to the slowest convergence time.

\subsection{A Coupon Collector automaton}

Our examination begins with the simple rule $\mathbb{T} 63$ :

\begin{tabular}{|c||c|c|c|c|c|c|}
\hline$s$ & 0 & 1 & 2 & 3 & 4 & 5 \\
\hline$f(s)$ & 1 & 1 & 1 & 1 & 1 & 1 \\
\hline
\end{tabular}

An update sets the cell into state 1 whatever its initial state. The only fixed point is the all-1 configuration: $\mathfrak{F}_{63}=1^{\Lambda}$. It is reached in one step under the synchronous dynamics. In the asynchronous case, this fixed point is attained when each cell has been updated at least once (see Fig. 1). This kind of process often arises in the analysis of algorithms (see e.g., [5]) ; it is usually called a Coupon collector process.

\section{Theorem 1.}

$$
\mathrm{WECT}_{63}(n)=\Theta(n \log n)
$$




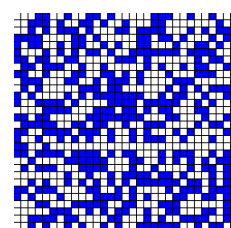

$t=0$

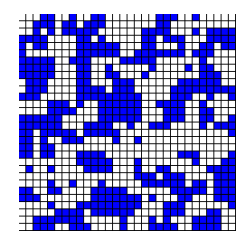

$t=n$

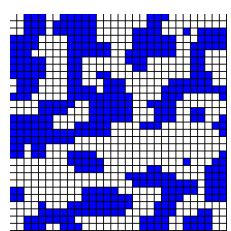

$t=3 n$

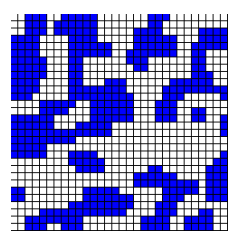

$t=17 n$

Figure 2: Example of a simulation for rule $\mathbb{T} 56$, when $\sigma^{0}$ is a random uniform configuration.

Proof. It is clear that the worst convergence time is attained with the configuration where all the cells are in state $0: \sigma^{0}=0^{\Lambda}$. We introduce $\mathcal{H}\left(\sigma^{t}\right)=\left|\sigma^{t}\right|_{0}$. At each time step, either $\mathcal{H}$ remains unchanged or it decreases by one. Precisely

$$
\mathbb{P}\left(\Delta \mathcal{H}_{t}=-1 \mid \sigma^{t}\right)=\frac{\mathcal{H}(t)}{n} .
$$

where $\Delta Z_{t}$ denotes the increment of a function $Z$ at time $t$, that is, $\Delta Z_{t}=$ $Z\left(\sigma^{t+1}\right)-Z\left(\sigma^{t}\right)$. We keep this notation in the following of the article.

Thus

$$
T_{63}\left(\sigma^{0}\right)=\sum_{i=1}^{n} \mathcal{G}_{i},
$$

where $\left(\mathcal{G}_{i}\right)_{i \geq 1}$ is a sequence of independent r.v. with $\mathcal{G}_{i}$ a geometric r.v. of parameter $(n-i+1) n^{-1}$. We obtain

$$
\begin{aligned}
\mathbb{E}\left[T_{63}\left(\sigma^{0}\right)\right] & =\sum_{i=1}^{n} \mathbb{E}\left[\mathcal{G}_{i}\right]=\sum_{i=1}^{n} \frac{n}{n-i+1} \\
& =n H_{n}=\Theta(n \log n),
\end{aligned}
$$

( $H_{k}$ stands for the $k$-th harmonic number).

\subsection{The Majority rule}

We now turn to the Majority rule $\mathbb{T} 56$ :

\begin{tabular}{|c||c|c|c|c|c|c|}
\hline$s$ & 0 & 1 & 2 & 3 & 4 & 5 \\
\hline$f(s)$ & 0 & 0 & 0 & 1 & 1 & 1 \\
\hline
\end{tabular}

An update on cell $c$ sets this cell to the state found in majority in its neighbourhood $\mathcal{N}(c)$. The global effect of the rule is to converge quickly to an equilibrium where homogeneous regions of 0 s and 1s coexist (see Fig. 2). 


\section{Theorem 2.}

$$
\mathrm{WECT}_{56}(n)=\Theta\left(n^{2}\right)
$$

To prove the theorem, we introduce the following Lemma [4]:

Lemma 1. Let $\tau$ be a stopping time with regard to a filtration $\left(\mathcal{F}_{t}\right)$ and $\left(S_{t}\right)$ a sequence of positive random variables adapted to $\left(\mathcal{F}_{t}\right)$, with $S_{0}=s_{0}$. Assume that there exists $\varepsilon>0$ such that, for $t<\tau$,

$$
\mathbb{E}\left[\Delta S_{t} \mid \mathcal{F}_{t}\right] \leq-\varepsilon
$$

then

$$
\mathbb{E}[\tau] \leq \frac{s_{0}}{\varepsilon} .
$$

Intuitively, a filtration represents the information gained by an observation of the process. For the sake of simplicity, we will not define it formally here, we will instead simply consider that there exists a natural filtration $\mathcal{F}_{t}$ associated to the sequence of configuration $\left(\sigma^{t}\right)$.

The proof of Theorem 2 consists of two parts: the upper bound on the WECT is obtained by considering an energy function, the lower bound by analysing the convergence of a particular configuration.

Proof. We introduce $\mathcal{I}$, an anti-similarity function that counts the number of pairs of neighbouring cells in different states:

$$
\mathcal{I}(\sigma)=\operatorname{card}\left\{\left\{c, c^{\prime}\right\} \in \Lambda^{2} \mid d\left(c, c^{\prime}\right)=1 \text { and } \sigma_{c} \neq \sigma_{c^{\prime}}\right\} .
$$

We obtain an upper bound on the convergence time by showing that the sequence $\mathcal{I}\left(\sigma^{t}\right)$ is decreasing. To see why this holds, first note that if an updating changes the state of the cell, then one of the four cases below has occurred, and then $\mathcal{I}$ has decreased by at least 2 .

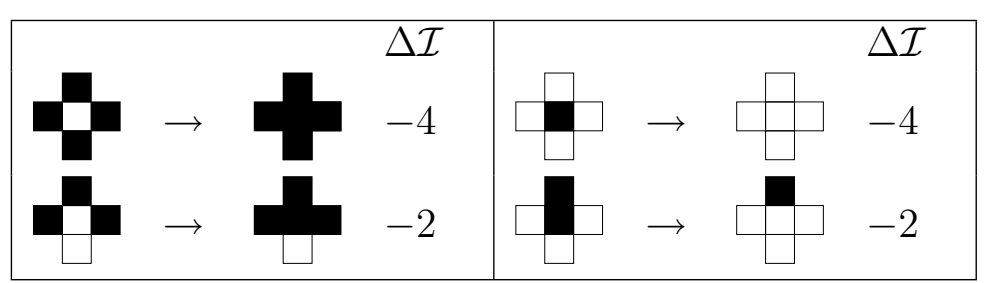

Thus, for $t<T$,

$$
\mathbb{E}\left[\Delta I_{t} \mid \sigma^{t}\right] \leq-\frac{2}{n}
$$


Using Lemma 1 with $S_{t}=I\left(\sigma^{t}\right)$, as $\mathcal{I}$ takes its values in $\{0, \ldots, 2 n\}$, we have that for any initial configuration $\sigma^{0}$,

$$
\mathbb{E}\left[T_{f}\left(\sigma^{0}\right)\right] \leq \frac{n}{2} I\left(\sigma^{0}\right) \leq n^{2} .
$$

Lower bound. To obtain a lower bound on the convergence time, we consider the snake-like initial configuration $\sigma_{\mathrm{sn}}$ represented below (for $L=10$ ):

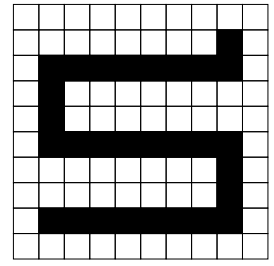

Intuitively, this initial configuration is constructed in order to ensure that the only way of reaching equilibrium is to "shrink" the snake by updating its two extremities. As the probability to update one of the two extremities is $2 / n$, we obtain

$$
\mathbb{E}\left[T_{56}\left(\sigma_{\mathrm{sn}}\right)\right] \geq \sum_{i=1}^{\left|\sigma_{\mathrm{sn}}\right|_{1}-1} \mathbb{E}\left[\mathcal{G}_{i}\right]
$$

where $\mathcal{G}_{i}$ 's are i.i.d. geometric r.v. with parameter $2 / n$. The snake is made of about $n / 3$ cells in state 1 ; as we have $\left|\sigma_{\mathrm{sn}}\right|_{1} \sim n / 3$,

$$
\mathbb{E}\left[T_{56}\left(\sigma_{\mathrm{sn}}\right)\right] \geq \mathrm{C}^{\mathrm{st}} n^{2} .
$$

Remark 1. It is easy to see that the number of fixed points of $\mathbb{T} 56$ is exponential in $n$. To describe more precisely the set of fixed points of the majority rule $\mathfrak{F}_{56}$, we may note that fixed points correspond to the local minima of the "energy" function $\mathcal{I}$.

\subsection{The Epidemic automaton}

Up to now, the classes of convergence we met were already known for $1 \mathrm{D}$ cellular automata [3]. Let us consider the Epidemic rule $\mathbb{T} 62$, which convergence type is new and specific to the two-dimensional case:

\begin{tabular}{|c||c|c|c|c|c|c|}
\hline$s$ & 0 & 1 & 2 & 3 & 4 & 5 \\
\hline$f(s)$ & 0 & 1 & 1 & 1 & 1 & 1 \\
\hline
\end{tabular}




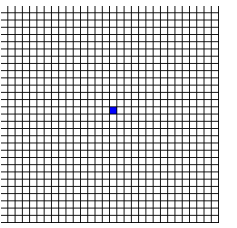

$t=0$

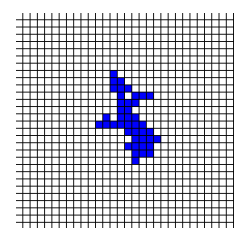

$t=2 n$

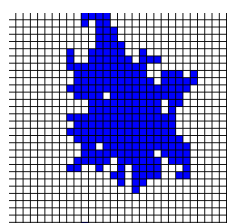

$t=5 n$

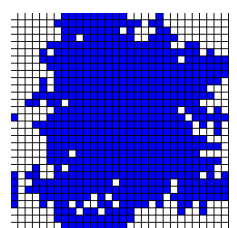

$t=8 n$

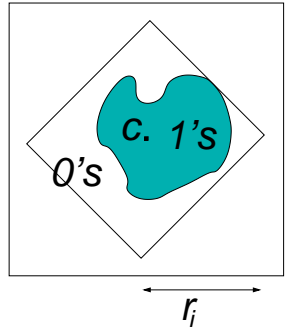

Figure 3: Top : Example of a simulation for rule $\mathbb{T} 62$. Bottom : Schematic view of a configuration where a region of 1 's reaches the sphere $\partial\left(c, r_{i}\right)$.

A cell in state 0 (healthy) turns to state 1 (infected) if one of its neighbours is in state 1 ; it then remains in this state for ever. The global effect of this rule is to make the connected components of $1 \mathrm{~s}$, the 1-regions, grow and fill the whole grid $\Lambda$.

Theorem 3. There exist two constants $C^{-}, C^{+}$such that, for n large enough,

$$
\frac{C^{-}}{\log n} n^{3 / 2} \leq \operatorname{WECT}_{62}(n) \leq C^{+}(\log n) n^{3 / 2} .
$$

Remark 2. We conjecture that one may tighten these bounds and show that $\mathrm{WECT}_{62}(n)$ is actually of order $n^{3 / 2}$.

Remark 3. Our problem is very close to that introduced by Eden [1], except that in our model, changes of state occur at random times. Richardson [8] has shown that the 1-region $\mathfrak{R}_{1}$ in the Eden model admits an asymptotic shape. Informally, his result may be stated as follows: with infinite environment,

$$
\mathfrak{R}_{1} / \operatorname{Card}\left(\mathfrak{R}_{1}\right) \rightarrow C,
$$

where the fraction denotes a spatial rescaling operation and where $C$ is a deterministic convex subset of $\mathbb{R}^{2}$. This result does not tell us however at which speed the 1-region $\mathfrak{R}_{1}$ grows.

Proof. The first step is to prove that the largest convergence time is obtained for the configurations which contain only one cell in state 1 . Let us introduce a partial order $\prec$ on $\{0,1\}^{\Lambda}$ by:

$$
\forall(\sigma, \eta) \in \Lambda^{2}, \quad \sigma \prec \eta \text { if and only if } \forall c \in \Lambda, \sigma_{c} \leq \eta_{c} .
$$


It suffices to note that $\Phi$ conserves the order $\prec$ in the sense that:

$$
\forall U_{t} \in \Lambda, \sigma \prec \eta \Rightarrow \Phi\left(\sigma, U_{t}\right) \prec \Phi\left(\eta, U_{t}\right)
$$

We therefore obtain that for any two configurations $\sigma$ and $\eta$ such that $\sigma \prec \eta$, $\sigma^{t}=1^{\Lambda} \Rightarrow \eta^{t}=1^{\Lambda}$; which proves the first step. The second step is to establish the two bounds in Theorem 3 when $\sigma^{0}$ contains one single cell in state 1.

Lower bound. Let us denote by $c$ the only 1 in the initial configuration $\sigma^{0}$. The idea for establishing a lower bound is to "slice" the grid into concentric balls of center $c$, separated by a distance $r$. Let us denote by $\mathcal{B}_{l}$ the $l$-th ball, i.e., $\mathcal{B}_{l}=\partial B(c, r \times l)$ is the set of cells lying at distance l.r from $c$.

All along the process, the 1-region will grow, our objective is to find a lower bound on the time needed to go from the $(l-1)$-th ball to the $l$-th ball. To do this, we fix the value of $r$ to $r=\lceil\log L\rceil$ and for any $l$, let $\rho_{l}$ be the first time it reaches $\mathcal{B}_{l}$, namely

$$
\rho_{l}=\inf _{t \geq 0}\left\{\exists i \in \mathcal{B}_{l} \mid \sigma_{i}^{t}=1\right\}
$$

and set $\rho_{0}=0, \tau_{l}=\rho_{l}-\rho_{l-1}$. Then

$$
T_{62}\left(\sigma^{0}\right) \geq \tau_{1}+\tau_{2}+\cdots+\tau_{\lfloor L / 2 r\rfloor}
$$

where $T$ is the time of convergence. The upper bound follows now from:

Lemma 2. There exists a constant $C$ such that, for any $l \leq\lfloor L / 2 r\rfloor$,

$$
\mathbb{E}\left[\tau_{l}\right] \geq C \cdot L^{2} .
$$

Proof of the Lemma. Let us focus on a given $l$. A path joining $\mathcal{B}_{l-1}$ and $\mathcal{B}_{l}$ of length $j$ is a sequence of $j$ neighbour cells $\left(c_{1}, c_{2}, \ldots, c_{j}\right)$ joining $\mathcal{B}_{l-1}$ to $\mathcal{B}_{l}$ i.e., (a) $c_{1}$ is in $\mathcal{B}_{l-1}, c_{j}$ is in $\mathcal{B}_{l}$ and (b) for $i=1, \ldots, j-1, d\left(c_{i}, c_{i+1}\right)=1$. Below is a representation of a path in grey circles :

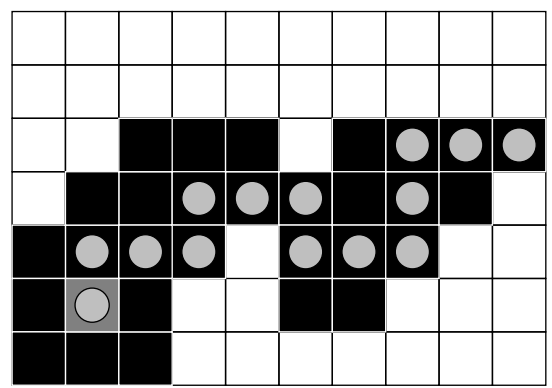


We first try to find an upper bound on $\mathbb{P}\left(\tau_{l} \leq k\right)$. If $\tau_{l}$ is less than $k$, then there exists a path joining $\mathcal{B}_{l-1}$ to $\mathcal{B}_{l}$ of length $j \geq r$, whose cells become 1 during the $k$ steps in the time interval from $\rho_{l-1}+1$ to $\rho_{l-1}+k$.

Let us denote by $\operatorname{Comp}(C, k, l)$ the event : "the cells of path $C$ were set to state 1 during the $k$ steps from $\rho_{l-1}+1$ to $\rho_{l-1}+k "$. We have that :

$$
\mathbb{P}\left(\tau_{\ell} \leq k\right) \leq \mathbb{P}\left(\bigcup_{C \in \mathcal{C}} \operatorname{Comp}(C, k, l)\right)
$$

where $\mathcal{C}$ denotes the set of paths joining $\mathcal{B}_{l-1}$ and $\mathcal{B}_{l}$ with a length smaller or equal than $k$. We can now split the calculus with regard to the sets $\left(C_{j}\right)$, where $\mathcal{C}_{j}$ is the set of paths of length $j$ :

$$
\mathbb{P}\left(\tau_{\ell} \leq k\right) \leq \sum_{j=r}^{k} \mathbb{P}\left(\bigcup_{C \in \mathcal{C}_{j}} \operatorname{Comp}(C, k, l)\right)
$$

A path in $\mathcal{C}_{j}$ starts from one of the $8(l-1) r$ cells of $\mathcal{B}_{l-1}$ and performs $j$ steps with 3 directions available at each step. We thus have card $\mathcal{C}_{j} \leq 8(l-1) r 3^{j}$.

On the other hand, for a path $C=\left(c_{1}, \ldots, c_{j}\right) \in \mathcal{C}_{j}$, let us denote by $U_{1}, \ldots, U_{k}$ be the $k$ random variables that have updated the cells in the time interval $\rho_{l-1}+1$ to $\rho_{l-1}+k$. There exist $j$ time steps $\left\{t_{1}, \ldots, t_{j}\right\}$ such that

$$
\left\{U_{t_{1}}, \ldots, U_{t_{j}}\right\}=\left\{c_{1}, \ldots, c_{j}\right\}
$$

We thus have that, for any path $C$ :

$$
\mathbb{P}(\operatorname{Comp}(C, k, l)) \leq\left(\begin{array}{c}
k \\
j
\end{array}\right) \frac{j !}{n^{j}} .
$$

From this inequality, we derive

$$
\begin{aligned}
\mathbb{P}\left(\tau_{l} \leq k\right) & \leq \sum_{j=r}^{k} 8(l-1) r 3^{j}\left(\begin{array}{c}
k \\
j
\end{array}\right) \frac{1}{L^{2 j}} \\
& \leq 8(l-1) r \sum_{j=r}^{k}\left(\begin{array}{l}
k \\
j
\end{array}\right) \frac{3^{j}}{L^{2 j}}
\end{aligned}
$$

Now, we remark that fixing $k \leq L^{2} / 10$ gives

$$
\begin{aligned}
\mathbb{P}\left(\tau_{l} \leq k\right) & \leq 4 L \sum_{j=r}^{k}(3 / 10)^{j} \text { as }(l-1) r \leq L / 2 \\
& \leq 4 L(3 / 10)^{r} .2 \leq 8 \exp (\log L(1+\log (3 / 10))),
\end{aligned}
$$


which tends to zero, as $1+\log (3 / 10)$ is negative. For our purpose, it is sufficient to bound this probability by $1 / 2$ (for large $L$ ). As we have $\mathbb{P}\left(\tau_{l}>\right.$ $k)=1-\mathbb{P}\left(\tau_{l} \leq k\right)$, it follows that

$$
\mathbb{E}\left[\tau_{l}\right] \geq \sum_{k=0}^{L^{2} / 9} \mathbb{P}\left(\tau_{l}>k\right) \geq \frac{L^{2}}{10} \cdot \frac{1}{2}
$$

We now conclude the proof for the lower bound with:

$$
\begin{aligned}
T_{62}\left(\sigma^{0}\right) & \geq \sum_{l=1}^{\lfloor L / 2 r\rfloor} \mathbb{E}\left[\tau_{l}\right] \\
& \geq C(L / \log L) L^{2} .
\end{aligned}
$$

Upper bound. This bound is easier to obtain. Contrary to the proof of the lower bound, we now consider the time $\varsigma_{l}$ at which all the cells in $\mathcal{B}_{l}$ are in state 1 . Set $\varsigma_{0}=0$, and $\tau_{l}=\varsigma_{l}-\varsigma_{l-1}$. Since the radius of the grid of size $L$ is $\lfloor L / 2\rfloor$,

$$
T_{62}\left(\sigma^{0}\right)=\tau_{1}+\cdots+\tau_{\lfloor L / 2\rfloor} .
$$

Lemma 3. There exists a constant $\lambda$, independent of $l$ and $L$, such that

$$
\mathbb{E}\left[\tau_{l}\right] \leq \lambda L^{2} \log l
$$

Proof of the Lemma. At time $\varsigma_{l-1}$, all the cells at distance $l-1$ are in state 1 , and so all the cells in $\mathcal{B}_{l}$ have a 1-neighbour, so it will be all-1 at the first time at which its $4 l$ cells have been updated. Thus, similarly to the proof for the Coupon Collector automaton,

$$
\begin{aligned}
\mathbb{E}\left[\tau_{l}\right] & \leq \mathbb{E}\left[\mathcal{G}_{4 l}+\cdots+\mathcal{G}_{1}\right], \\
& \leq \sum_{i=1}^{4 l} \frac{L^{2}}{i} \leq \lambda L^{2} \log l .
\end{aligned}
$$

Using Lemma 3, it follows that

$$
\mathbb{E}\left[T_{62}\left(\sigma^{0}\right)\right] \leq \sum_{l=1}^{L / 2} \lambda L^{2} \log l \leq \lambda_{2} L^{3} \log L \sim n^{3 / 2} \log n .
$$



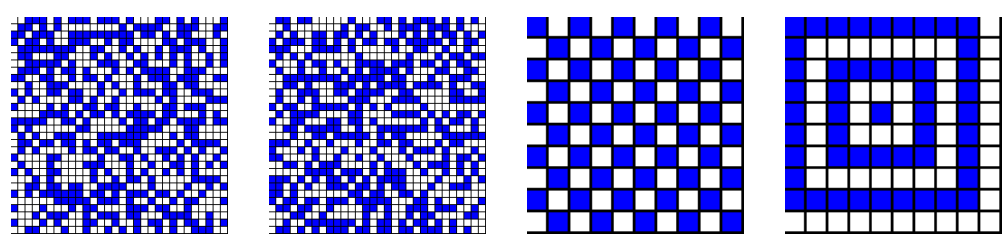

Figure 4: Left: Two typical noise-like configurations observed in the evolution of the erratic rule $\mathbb{T} 10$. Right:: Two fixed points of this rule.

\section{4 (Very) Slow convergence?}

We now examine two rules that illustrate how we may use analysis to distinguish between the "very long" and the infinite convergence times.

\subsection{Metastability for an Erratic automaton}

When exploring systematically the 64 totalistic rules, we observed that some of them evolved in a metastable regime: the observation of the configurations attained gives the impression that the system evolves "erratically" and will never converge. However, a detailed analysis show that these rules do possess fixed points and that some of them may be attained with a convenient sequence of updates. Among these rules, we focus on rule $\mathbb{T} 10$ :

\begin{tabular}{|c||c|c|c|c|c|c|}
\hline$s$ & 0 & 1 & 2 & 3 & 4 & 5 \\
\hline$f(s)$ & 0 & 1 & 0 & 1 & 0 & 0 \\
\hline
\end{tabular}

Figure 4 shows an example of noise-like configurations and examples of fixed points of rule $\mathbb{T} 10$. Hence, we can show that $\mathrm{WECT}_{10}(n)<+\infty$.

Conjecture 1. There exists a constant $\lambda>1$ such that, for $n$ large enough,

$$
\lambda^{n} \leq \mathrm{WECT}_{10}(n)<+\infty .
$$

Remark 4. This conjecture is consistent with the simulations, since we cannot observe convergence, except for very small grids (i.e., $n=4 \times 4$ or $6 \times 6$ ).

In [3], authors proved that there exist 1D CA for which the worst convergence time is exponential in $n$. It seems however that the methods used in dimension one do not apply here. It is an open problem to establish a proof of convergence for rule 10 or any other $2 \mathrm{D}$ rule that displays metastability. 


\subsection{The Parity Counter}

We finish our exploration of the 64 totalistic rules with rule $\mathbb{T} 42$ :

\begin{tabular}{|c||l|l|l|l|l|l|}
\hline$s$ & 0 & 1 & 2 & 3 & 4 & 5 \\
\hline$f(s)$ & 0 & 1 & 0 & 1 & 0 & 1 \\
\hline
\end{tabular}

It is sometimes referred to as the Parity Counter: an update on cell $c$ turns it into a 1 if and only if the number of 1 's in $N(c)$ is odd.

Theorem 4. If $\sigma^{0}$ is not a fixed point, then $T_{42}\left(\sigma^{0}\right)=+\infty$.

Consequently,

$$
\mathrm{WECT}_{42}(n)=+\infty .
$$

Proof. Let $\sigma^{0}$ be any initial configuration but a fixed point. There at least one unstable cell. When one of these unstable cells is updated, for rule $\mathbb{T} 42$, one of these four transitions occurs:
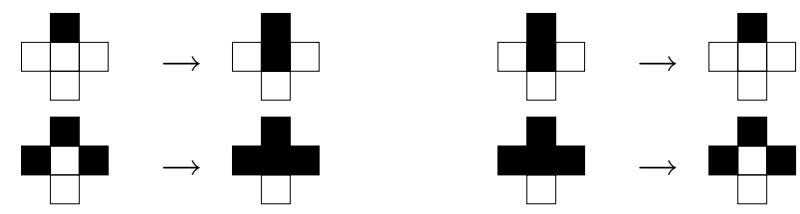

It is then easy to see that each transition is reversible, i.e., the updated cell stays unstable (but its neighbours might be stabilised).

Remark that the proof holds only for the case where a single cell is updated at each time step. For example, if we take two neighbouring 1-cells in a 0 grid, it becomes possible to converge to the fixed point $0^{\Lambda}$ if the two 1-cells are updated simultaneously:

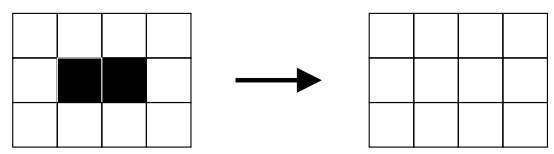

The parity counter thus provides a simple an interesting example that shows that a small difference in the updating function may trigger a qualitative change in the asymptotic behaviour of a cellular automaton. 


\subsection{Concluding remark}

According to our simulations, the spectrum of convergence times that we studied here covers most of the totalistic rules. We ask however if there are other convergence types. In particular, there are 8 rules $(e . g ., \mathbb{T} 7)$ for which we observe convergence to particular patterns such as checkerboards. In this case, we observe that boundaries between stable regions perform random walks. It is an open problem to determine the convergence time of these rules.

Table 1 gives a synthetic view of the 64 totalistic rules. Another challenging problem is to discriminate, among the rules for which we do not observe convergence, which ones evolve in a metastable state and which ones are truly non-converging.

Acknowledgements. Authors are grateful to P.-Y. Louis for enlightening discussions, and to M. Krikun for his comments on a first version of this paper. N. Fatès acknowledges the support of the INRIA ARC AMYBIA project.

\section{References}

[1] M. Eden. A two dimensional growth process. In Neyman J., editor, Proceeding of the Fourth Berkeley Symposium on Mathematical Statistics and Probability, volume 4, pages 222-239, Berkeley, University of California Press, 1961.

[2] Nazim Fatès. Fiatlux CA simulator in Java. See http://nazim.fates. free.fr for downloading.

[3] Nazim Fatès, Michel Morvan, Nicolas Schabanel, and Eric Thierry. Fully asynchronous behavior of double-quiescent elementary cellular automata. Theoretical Computer Science, 362:1-16, 2006.

[4] G. Fayolle, V.A. Malyshev, and M.V. Menshikov. Topics in the constructive theory of countable Markov chains. Cambridge University Press, 1995.

[5] Philippe Flajolet and Robert Sedgewick. An Introduction to the Analysis of Algorithms. Addison Wesley, 1986. 


\begin{tabular}{|c|c|c|c|c|c|}
\hline $\mathrm{CA}$ & 543210 & $\mathrm{CA}$ & 543210 & WECT & Observed behaviour \\
\hline $\mathbb{T} 0$ & 000000 & $\mathbb{T} 63$ & 111111 & $\Theta(n \log n)($ Coupon $)$ & Fast and monotonous conv. \\
\hline $\mathbb{T} 1$ & 000001 & $\mathbb{T} 31$ & 011111 & $+\infty$ (no fixed p.) & No observed conv. \\
\hline $\mathbb{T} 2$ & 000010 & $\mathbb{T} 47$ & 101111 & & Fast conv. with random walks \\
\hline $\mathbb{T} 3$ & 000011 & $\mathbb{T} 15$ & 001111 & & Fast conv. with random walks \\
\hline $\mathbb{T} 4$ & 000100 & $\mathbb{T} 55$ & 110111 & & Fast and monotonous conv. \\
\hline $\mathbb{T} 5$ & 000101 & $\mathbb{T} 23$ & 010111 & & No observed conv. \\
\hline $\mathbb{T} 6$ & 000110 & $\mathbb{T} 39$ & 100111 & & Fast conv. with random walks \\
\hline $\mathbb{T} 7$ & 000111 & $\mathbb{T} 7$ & 000111 & (Minority) & Fast conv. with random walks \\
\hline $\mathbb{T} 8$ & 001000 & $\mathbb{T} 59$ & 111011 & & Fast and monotonous conv. \\
\hline $\mathbb{T} 9$ & 001001 & $\mathbb{T} 27$ & 011011 & & No observed conv. \\
\hline $\mathbb{T} 10$ & 001010 & $\mathbb{T} 43$ & 101011 & $\geq \lambda^{n}$ (conjectured $)$ & No observed conv. \\
\hline $\mathbb{T} 11$ & 001011 & $\mathbb{T} 11$ & 001011 & & No observed conv. \\
\hline $\mathbb{T} 12$ & 001100 & $\mathbb{T} 51$ & 110011 & & No observed conv. \\
\hline $\mathbb{T} 13$ & 001101 & $\mathbb{T} 19$ & 010011 & & No observed conv. \\
\hline $\mathbb{T} 14$ & 001110 & $\mathbb{T} 35$ & 100011 & & Fast conv. with random walks \\
\hline $\mathbb{T} 16$ & 010000 & $\mathbb{T} 61$ & 111101 & & Fast and monotonous conv. \\
\hline $\mathbb{T} 17$ & 010001 & $\mathbb{T} 29$ & 011101 & & No observed conv. \\
\hline $\mathbb{T} 18$ & 010010 & $\mathbb{T} 45$ & 101101 & & No observed conv. \\
\hline $\mathbb{T} 20$ & 010100 & $\mathbb{T} 53$ & 110101 & & Fast and monotonous conv. \\
\hline $\mathbb{T} 21$ & 010101 & $\mathbb{T} 21$ & 010101 & & No observed conv. \\
\hline $\mathbb{T} 22$ & 010110 & $\mathbb{T} 37$ & 100101 & & No observed conv. \\
\hline $\mathbb{T} 24$ & 011000 & $\mathbb{T} 57$ & 111001 & & No observed conv. \\
\hline $\mathbb{T} 25$ & 011001 & $\mathbb{T} 25$ & 011001 & & No observed conv. \\
\hline $\mathbb{T} 26$ & 011010 & $\mathbb{T} 41$ & 101001 & & No observed conv. \\
\hline $\mathbb{T} 28$ & 011100 & $\mathbb{T} 49$ & 110001 & & No observed conv. \\
\hline $\mathbb{T} 30$ & 011110 & $\mathbb{T} 33$ & 100001 & & No observed conv. \\
\hline $\mathbb{T} 32$ & 100000 & $\mathbb{T} 62$ & 111110 & $\approx n^{3 / 2}$ (Epidemic) & Fast and monotonous conv. \\
\hline $\mathbb{T} 34$ & 100010 & $\mathbb{T} 46$ & 101110 & & Fast conv. with random walks \\
\hline $\mathbb{T} 36$ & 100100 & $\mathbb{T} 54$ & 110110 & & Fast and monotonous conv. \\
\hline $\mathbb{T} 38$ & 100110 & $\mathbb{T} 38$ & 100110 & & Fast conv. with random walks \\
\hline $\mathbb{T} 40$ & 101000 & $\mathbb{T} 58$ & 111010 & & Fast and monotonous conv. \\
\hline $\mathbb{T} 42$ & 101010 & $\mathbb{T} 42$ & 101010 & $+\infty$ (Parity) & No observed conv. \\
\hline $\mathbb{T} 44$ & 101100 & $\mathbb{T} 50$ & 110010 & & No observed conv. \\
\hline $\mathbb{T} 48$ & 110000 & $\mathbb{T} 60$ & 111100 & & Fast and monotonous conv. \\
\hline $\mathbb{T} 52$ & 110100 & $\mathbb{T} 52$ & 110100 & & Fast conv. with random walks \\
\hline $\mathbb{T} 56$ & 111000 & $\mathbb{T} 56$ & 111000 & $\Theta\left(n^{2}\right)$ (Majority) & Fast and monotonous conv. \\
\hline
\end{tabular}

Table 1: Synthesis of the 64 totalistic rule. Each rule is presented with its symmetric according to the $0 / 1$ symmetry. 
[6] Carlos Grilo and Luís Correia. Asynchronous stochastic dynamics and the spatial prisoner's dilemma game. In José Neves, Manuel Filipe Santos, and José Machado, editors, Progress in Artificial Intelligence, 13th Portuguese Conference on Aritficial Intelligence, EPIA 200\%, volume 4874 of Lecture Notes in Computer Science, pages 235-246. Springer, 2007.

[7] Damien Regnault, Nicolas Schabanel, and Eric Thierry. Progresses in the analysis of stochastic $2 \mathrm{~d}$ cellular automata: A study of asynchronous $2 \mathrm{~d}$ minority. In Ludek Kucera and Antonín Kucera, editors, Mathematical Foundations of Computer Science 2007, 32nd International Symposium, MFCS 2007, volume 4708 of Lecture Notes in Computer Science, pages 320-332. Springer, 2007.

[8] Richardson. Random growth in a tessellation. Mathematical Proceedings of the Cambridge Philosophical Society, 74:515-528, 1973. 